\title{
Índices de estabilidad de voltaje calculados a partir del flujo de potencia continuado
}

\author{
Johonel Caceres Espinoza \\ Johonel.caceres@unsaac.edu.pe \\ Universidad Nacional de San Antonio Abad del Cusco \\ Lucio Caceres Espinoza \\ lcaceres@untels.edu.pe \\ Universidad Nacional Tecnológica de Lima Sur
}

\section{RESUMEN}

El propósito de esta investigación consiste en determinar los índices de estabilidad de voltaje, ante perturbaciones que puedan ocurrir dentro de un sistema eléctrico de potencia (incrementos de la demanda), los cuales son causantes de factores críticos, que alteran la operación de un sistema eléctrico de potencia, debido a que provocan la salida intempestiva de los elementos de transmisión y generación (accionamiento de los sistemas de protección) por disminución de los niveles de voltaje. Se utilizará el método de parametrización geométrica para la determinación de los índices de estabilidad, debido a que facilita el cálculo del vector de estado (módulos y ángulos de voltaje) y la eliminación de la singularidad de la matriz Jacobiana, utilizados en la evaluación de la inestabilidad de voltaje. Asimismo, se aplicará dicha metodología al sistema de prueba de la IEEE de 14 barras, con ayuda de un algoritmo programado en Matlab.

Palabras clave: índices de estabilidad; pérdidas de potencia; matriz jacobiana; singularidad de matrices. 


\title{
Voltage stability indices calculated from continuous power flow
}

\begin{abstract}
The purpose of this research is to determine the voltage stability indices, in the face of disturbances that may occur within an electrical power system (increases in demand), which are the cause of critical factors that alter the operation of an electrical system. of power, because they cause the untimely output of the transmission and generation elements (actuation of the protection systems) due to the decrease in voltage levels. The geometric parametrization method will be used to determine the stability indices, because it facilitates the calculation of the state vector (modules and voltage angles) and the elimination of the singularity of the Jacobian matrix, used in the evaluation of the voltage instability. Likewise, this methodology will be applied to the IEEE 14-bar test system, with the help of an algorithm programmed in Matlab.
\end{abstract}

Keywords: stability indices; power losses; Jacobian matrix; matrix singularity

Artículo recibido: 05 octubre. 2021 Aceptado para publicación: 02 noviembre 2021 Correspondencia: Johonel.caceres@unsaac.edu.pe Conflictos de Interés: Ninguna que declarar 


\section{INTRODUCCIÓN}

El crecimiento de los sistemas eléctricos de potencia, debido al incremento de la demanda de energía eléctrica, genera la necesidad de tener un mayor número de interconexiones entre los diversos sistemas de generación - carga. De esta manera, los sistemas interconectados son ventajosos en la medida que permiten atender a los usuarios con un menor costo de operación. Asimismo, el mallar la red conduce al uso eficiente del parque de generación, con el cual se puede atender variaciones de cargas súbitas, compra y venta de energía y un mejor aprovechamiento de las diversidades hidrológicas existentes.

Desafortunadamente, aun se cuenta con un déficit de elementos de transmisión, sistemas interconectados, que se encuentran en sus límites de operación, produciendo grandes pérdidas de energía y demanda no atendidas. Asimismo, cualquier incremento sustancial de la demanda, ocasionaría una disminución en los márgenes de operación (cargabilidad), al igual que una disminución de los niveles de voltaje en las barras de un sistema eléctrico de potencia, llevando muchas veces al sistema, a una inestabilidad de voltaje (caracterizado por una caída progresiva en los módulos de voltaje) y al accionamiento de los equipos de protección, interrumpiendo de esta manera, el continuo servicio y generando grandes pérdidas por falta de energía.

Es por ello, que se hace necesario el cálculo de los índices de estabilidad de voltaje, debido a que se obtendrán los márgenes de seguridad de voltaje y las cargabilidades de los elementos de transmisión (capacidad de soporte de potencia) ante incrementos de la demanda, de manera que se obtenga una respuesta rápida de la operación ante este tipo de perturbaciones.

Cabe mencionar, que el cálculo de los índices de estabilidad de voltaje (que lo hace más difícil de entender y solucionar), es que es un fenómeno no lineal y cuanto mayor sea la cantidad de componentes (elementos de transmisión y transformación), mayor será la dificultad de obtener los parámetros que definen la operación de un sistema eléctrico de potencia (módulos y ángulos de voltaje de las barras del sistema), es por ello, la utilización de un método que sea versátil y practico, como es el caso de la utilización de métodos geométricos, que eliminan la singularidad de las matrices que modelan un sistema eléctrico de potencia, hasta llegar al grafico de la región de inestabilidad. 


\section{ESTRATEGIAS METODOLÓGICAS O MATERIALES Y MÉTODOS}

\subsection{Parametrización Geométrica, para el Cálculo de Flujo de Potencia Continuo}

Este método consiste, en incrementar la demanda de un sistema interconectado (partiendo de un caso base), y verificar como disminuye progresivamente el voltaje de las barras del sistema. Cabe mencionar que se llegará a un límite de incremento de demanda (determinado margen de cargabilidad), donde se obtendrá un punto de colapso de voltaje (o punto de máxima cargabilidad PMC) donde el voltaje puede disminuir rápidamente (región de inestabilidad de voltaje) y el sistema deja de funcionar.

Para la aplicación de este método, se debe incrementar un nuevo sistema de ecuaciones, a las ecuaciones de flujo de potencia (ecuaciones que determinan la operación de un sistema eléctrico de potencia), según se describe en la ecuación (1), donde dicha ecuación representa una línea recta, que pasa por un punto escogido $O\left(\lambda^{0}, V^{0}\right)$ inicial, en el plano de voltaje - cargabilidad (plano $V-\lambda$ ), con el fin de eliminar la singularidad de la matriz que define la operación de un sistema eléctrico de potencia

$$
W(\theta, V, \lambda, \alpha)=\alpha\left(\lambda-\lambda^{0}\right)-\left(V_{k}-V_{k}^{0}\right)=0
$$

\section{Donde:}

- $\lambda$ es el factor de cargabilidad, medido en pu (sistema en por unidad)

- $V_{k}$ es la magnitud del voltaje de la barra bajo estudio, medido en pu (sistema en por unidad)

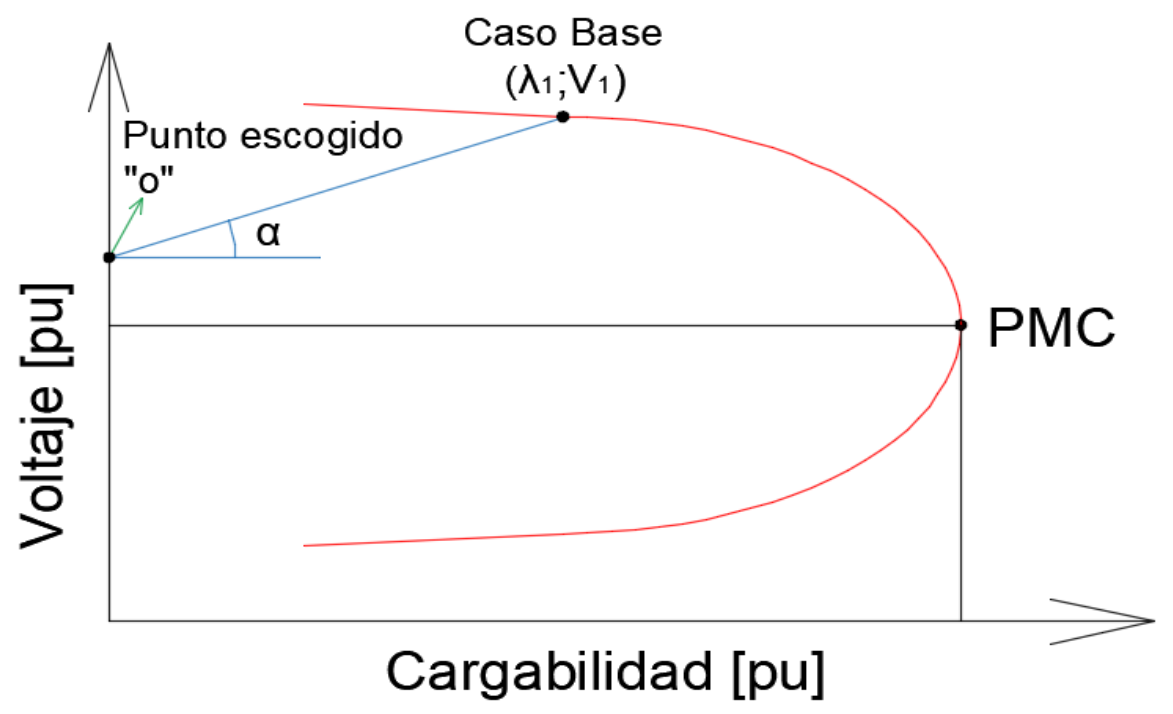

Figura 1 - Grafica del voltaje en Función de la Cargabilidad. 
Además, el parámetro $\alpha$ es un coeficiente angular que disminuirá en un porcentaje constante, para crear n puntos de operación en los sistemas de transmisión. Como, una nueva ecuación es adicionada, $\lambda$ puede ser tratado como una variable dependiente y $\alpha$ como una variable independiente (pendiente de una recta), siendo este valor escogido, como un parámetro de continuación (su valor es fijado). Así mismo, el número de incógnitas es igual al número de ecuaciones, esto es una condición necesaria para que se obtenga una solución al sistema de ecuaciones y de esta manera hace que la matriz Jacobiana (J) no sea singular.

Con la solución, de las variables de estado, del caso base $\left(\theta^{1}, V^{1}\right.$ y $\left.\lambda^{1}\right)$ obtenida a partir de un flujo de potencia, se procede al cálculo del primer coeficiente angular $\alpha^{1}$ a partir de un punto inicial escogido $O\left(\lambda^{0}, V^{0}\right)$ y de los respectivos valores obtenidos en el caso base $P\left(\lambda^{1}, V_{k}^{1}\right)$

$$
\alpha^{1}=\frac{V_{k}^{1}-V_{k}^{0}}{\lambda^{1}-\lambda^{0}}
$$

En seguida, el flujo de potencia continuo propuesto, se utiliza para calcular las demás soluciones a través de sucesivos decrementos $(\Delta \alpha)$ para el valor de $\alpha$. Para $\alpha=\alpha^{1}+\Delta \alpha$, la solución de las ecuaciones de flujo de potencia y la ecuación (1) de forma que el nuevo punto de operación $\left(\theta^{2}, V^{2}\right.$ y $\left.\lambda^{2}\right)$ correspondiente a la intersección de la trayectoria de soluciones (curva P-V) haciendo que el nuevo valor del coeficiente angular $\left(\alpha^{1}+\Delta \alpha\right)$ sea calculado y luego especificado. Para $\alpha=\alpha^{1}$, representará una solución de convergencia cuando $\lambda=1$. Una expansión al sistema de ecuaciones flujo de potencia tradicional y la ecuación (1) con respecto a la serie de Taylor, incluyendo solo los términos de primer orden y considerando un valor prefijado del valor del parámetro $\alpha$, el que fue calculado del caso base, resulta en:

$\left[\begin{array}{cc}-J_{a c} & -G_{\lambda} \\ -\partial W / \partial z & -\alpha\end{array}\right]\left[\begin{array}{l}\Delta z \\ \Delta \lambda\end{array}\right]=J_{m}\left[\begin{array}{l}\Delta z \\ \Delta \lambda\end{array}\right]=\left[\begin{array}{c}\Delta G \\ \Delta W\end{array}\right]$

\section{Dónde:}

- $z=\left[\begin{array}{ll}\theta & V\end{array}\right]^{T}$ es la matriz de las variables de estado

- $J_{a c}$ es la matriz Jacobiana tradicional de flujo de potencia.

- $G_{\lambda}$ corresponde a la derivada de $G$ en relación a factor de cargabilidad $\lambda$. 
Los valores de $\Delta G$ y $\Delta W$ representan los factores de corrección (vector de errores) de las ecuaciones de flujo de potencia y la ecuación (1). Se debe observar que estos valores deben ser cero (o prácticamente nulos, esto es con referencia a la tolerancia adoptada) para que se diga que el caso base convergió. Asimismo, el valor de $\Delta W$ será diferente de cero debido a las variaciones de $\alpha$ que a su vez son afectadas por el incremento de $\Delta \alpha$. Observe que las coordenadas del punto escogido $O\left(\lambda^{0}, V^{0}\right)$, mostrado en la Figura 1, puede ser movido a otro punto del plano, dependiendo entonces de la curva $P-V$ de la barra en estudio. La mudanza del punto escogido, es necesaria cuando se excede el número de iteraciones a un valor predefinido, o cuando el método diverge (análisis de errores). Un criterio para poder escoger el valor del punto escogido es que no exceda a un número base de iteraciones a lo largo de todo el trazado de la curva. Es importante destacar que en los métodos existentes en la literatura, la mudanza de parámetros a lo largo del trazado de la curva $P-V$ es necesaria, haciendo que la estructura de la matriz Jacobiana $\left(J_{a c}\right)$ sea modificada. En caso del método propuesto, es muy importante la mudanza de parámetros a lo largo de todo el trazado de la curva $P$ - $V$, la cual implica la mudanza de la matriz Jacobiana, en relación al valor del elemento, correspondiente a la derivada de $W$ respecto a $\lambda$ (valor conocido como $\alpha$ ).

\section{2. Índices Basados en los Niveles de Voltaje de cada Barra}

Uno de los parámetros que se debe mantener dentro de los márgenes de seguridad, es el módulo de voltaje de las barras, debido a que esta variable de estado, es la responsable de definir la operación de un sistema eléctrico de potencia, y determinar la estabilidad de voltaje, por lo que es de interés, el poder controlar este parámetro.

Podemos observar, que cuando se incrementa la demanda, los módulos de voltaje disminuye, llegando a una inestabilidad de voltaje, cuando se presentan súbitos incrementos de la demanda. Es por ello, que los niveles de voltajes, son considerados como buenos indicadores de estabilidad, pues también marcan el grado de capacidad, en la que se pueda entregar energía, antes de llegar al límite máximo de cargabilidad, donde el sistema deja de funcionar.

De acuerdo al método de parametrización geométrica, también podremos observar un comportamiento decreciente del voltaje, debido a un incremento progresivo de la demanda, este tipo de diagramas, ayudan a determinar hasta qué punto se puede entregar energía, antes de llegar a la inestabilidad de voltaje. 


\section{3. Índices Basados en las Pérdidas de Potencia Activa y Reactiva}

Este índice de estabilidad, determinar la cantidad de pérdidas que se puedan producir en los elementos de transmisión (líneas de transmisión y transformadores), cuando se incrementa la demanda, debido a que, si un sistema eléctrico produce grandes pérdidas, ocasionaría que el sistema se encuentre más cercano a la inestabilidad de voltaje.

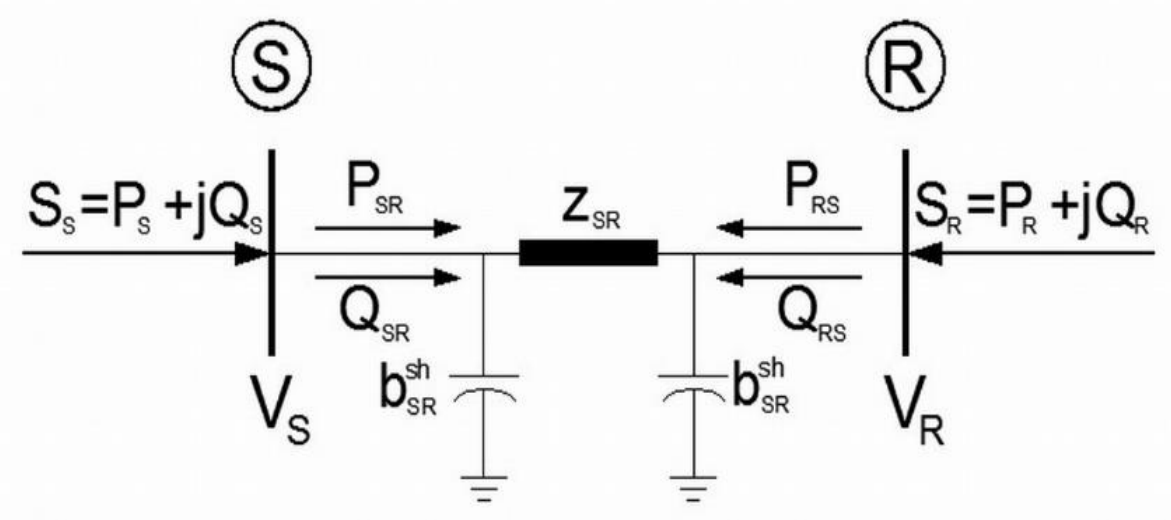

Figura 2 - Dirección de los Flujos de Potencia en una Línea de Transmisión

Para determinar este índice de estabilidad, se sumaría la cantidad de potencia activa y reactiva que se entrega en ambos puntos de conexión de un elemento de transmisión, donde la diferencia de ambos flujos, se considera la cantidad de pérdidas que se produce por transmisión de energía.
$\Delta P_{S R}=P_{S R}+P_{R S}$
Ec. 4
$\Delta Q_{S R}=Q_{S R}+Q_{R S}$
Ec. 5

\section{4. Índices Basados en los Flujos de Potencia Activa y Reactiva (Ipq)}

Este índice de estabilidad, se basa en la cantidad de potencia activa y reactiva que se transmite a través de los elementos de transmisión (líneas de transmisión y transformadores), donde a mayor cantidad de demanda, mayor será la cantidad de potencia que deba atravesar los elementos de transmisión y por consiguiente, disminuirá la capacidad de transporte, produciendo adicionalmente que la pérdidas se incrementen Para determinar este índice, utilizaremos la ecuación cuadrática del flujo de potencia, a través de una línea de transmisión, en función de los parámetros de estado, como son los módulos y ángulos de voltaje, de las barras de un sistema eléctrico de potencia, tal como se muestra en la ecuación 6. 


\section{Donde:}

- $A=\delta_{S R}$

- $U=V_{R}^{2}$

- $\quad a=1+z_{R S}^{2} b_{S R}^{s h^{2}}+2 z_{R S} b_{S R}^{s h} \operatorname{sen}(A)$

- $b=-a_{S R}{ }^{2} V_{S}{ }^{2}-2 z_{R S} P_{R S} \cos (A)+2 Q_{R S} z_{R S} \operatorname{sen}(A)+2 Q_{R S} Z_{R S} b_{S R}^{S h}$

- $c=z_{R S}^{2}\left(Q_{R S}^{2}+P_{R S}^{2}\right)$

Como se observa, la ecuación 6, representa una ecuación de segundo grado, que se puede solucionar mediante el método de bhaskara

$$
V_{R}= \pm \sqrt{\frac{-b \pm \sqrt{b^{2}-4 a c}}{2 a}}
$$

Para obtener una solución real, de este sistema de ecuaciones, se debe de considerar que el radical de como resultado valores reales y ello se cumple, siempre y cuando sea mayor a cero, tal como se muestra en la ecuación 8

$$
b^{2}-4 a c>0
$$

Estos valores reales, representan un índice de estabilidad $I_{p q}$, que muestran el grado de cargabilidad que se tiene en los elementos de transmisión por cantidad de potencia que se transmite a través de estos.

$$
I_{p q}=b^{2}-4 a c
$$

\section{RESULTADOS Y DISCUSIÓN}

Para el análisis de los índices de estabilidad, se utilizó el sistema de prueba de la IEEE de 14 barras, y el programa Matlab, debido a que constituye una herramienta útil, para el análisis de sistemas de potencia y por tener un alto grado de flexibilidad de simulación e implementación de algoritmos, para la solución de nuevas tecnologías. 


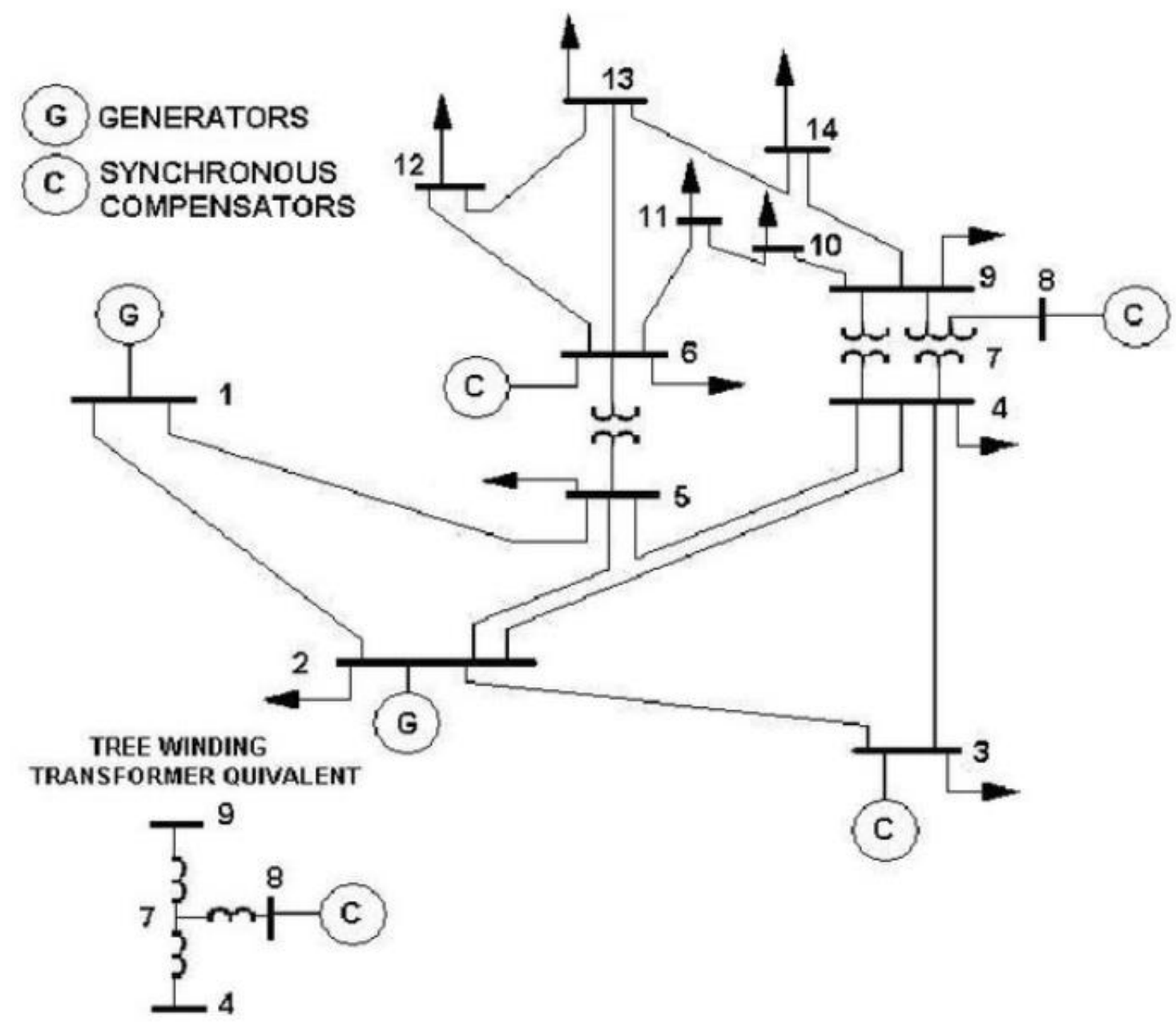

Figura 3 - Sistema de Prueba de la IEEE - 14 barras

De acuerdo a la Figura 4, se aplicó el método de parametrización geométrica, con el fin de obtener los márgenes de estabilidad de voltaje y los márgenes de cargabilidad del sistema de prueba, donde a incrementos de la demanda, en factores de por unidad (sistema adimensional) los voltajes en las 14 barras del sistema tienden a decrecer, llegando a valores mucho menores del margen de seguridad de voltaje. estas disminuciones, llegan a un punto (denominado Punto de Máxima cargabilidad), donde el sistema ya no funciona y las tensiones de las barras tienden a disminuir más rápidamente; esta concavidad inferior se considera la región inestable del sistema eléctrico de potencia.

Asimismo, el sistema solo puede soportar una cargabilidad del $173.86 \%$ de su demanda nominal (1.7386 pu), antes de llegar a un colapso de voltaje 


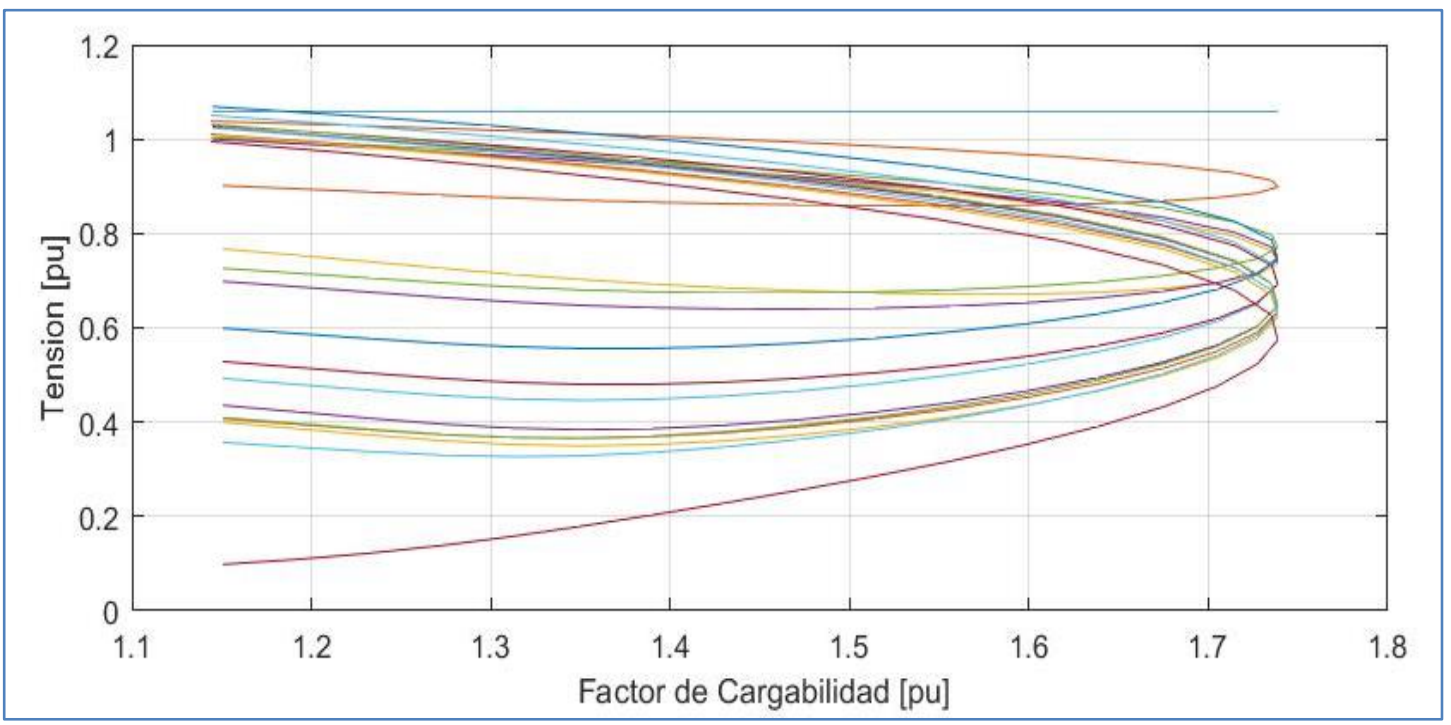

Figura 4 - Tendencia de los voltajes en las barras del Sistema IEEE de 14 barras ante incrementos del factor de cargabilidad

Respecto a la figura 5, se consideró como PLOSS, las pérdidas de potencia activa y QLOSS, las pérdidas de potencia reactiva, en los 20 elementos de transmisión, que conforma el sistema de prueba de la IEEE de 14 barras, donde se observa que a incremento de la demanda en valores por unidad (Lambda) las pérdidas tienden a crecer, teniendo un comportamiento exponencial creciente. Si bien, las pérdidas son consideradas variables de operación propias del sistema (dependencia de las variables de estado) tienen una relación directa con la estabilidad de voltaje, debido a que el sistema se encuentre más cargado, mayores serán las pérdidas de potencia activa y reactiva, respecto a la cantidad de potencia transmisión en los elementos de transmisión.

Adicionalmente, las máximas magnitudes de pérdidas son alcanzadas, cuando el sistema eléctrico llega hasta el punto de máxima cargabilidad (PCM) el cual representa el $173.86 \%$ de la demanda del sistema de prueba, teniendo como consecuencia, que el sistema deja de funcionar, una vez alcanzado el PCM.

En concordancia con la figura de las tendencias de voltaje, podemos indicar que las pérdidas del sistema, alcanzan en la misma magnitud de cargabilidad, por lo que el sistema llega a perder su estabilidad de voltaje.

Por otro lado, aquellos elementos de transmisión que logren alcanzar magnitudes de pérdidas elevadas, son los que contribuyen más, a la inestabilidad del sistema. 


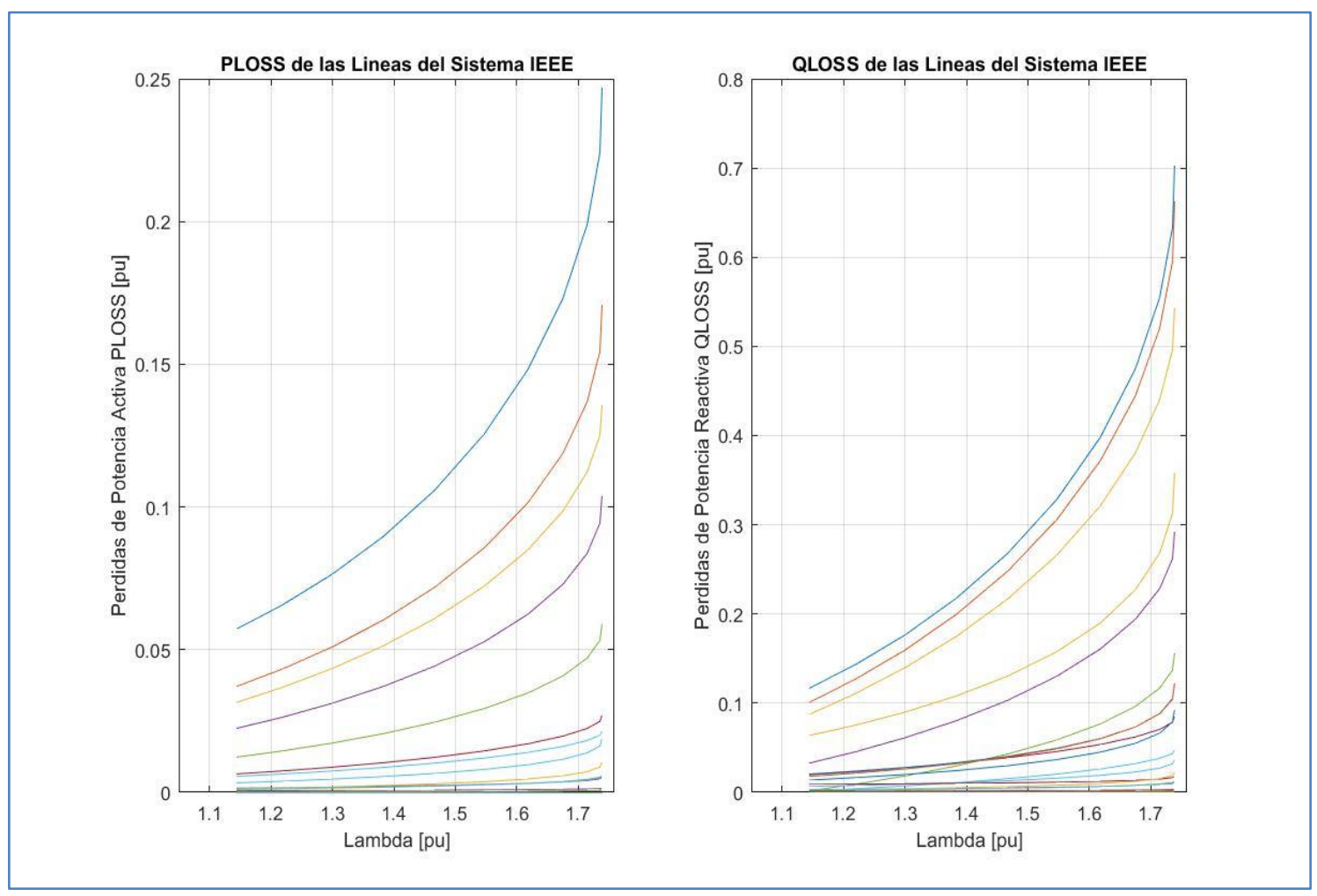

Figura 5 - (a) Comportamiento de las pérdidas de potencia activa ante incrementos del factor de cargabilidad para el Sistema IEEE de 14 Barras (b) Comportamiento de las pérdidas de potencia reactiva ante incrementos del factor de cargabilidad para el Sistema IEEE de 14 Barras

De la misma manera, el índice basado en los flujos de potencia activa y reactiva, se calcula a partir de los flujos de potencia, que atraviesan los elementos de transmisión. En la figura 6, se observa que las magnitudes de Ipq, puede tener valores negativos, y cuya tendencia es decreciente, esto respecto a incrementos de la demanda. Asimismo, en dicha simulación, se verifica, que aquellos elementos de transmisión que llegan a tener valores negativos, son considerados los elementos más cargados, por lo que su contribución a la inestabilidad es mayor, mientras que las líneas de transmisión que obtengan valores solamente valores positivos y con magnitudes elevadas, son considerados elementos robustos, que pueden llegar a soportar estos incrementos de la demanda.

Los valores Ipq, alcanzan sus valores mínimos, cuando la cargabilidad alcanzada es del $173.86 \%$ de la demanda del sistema de prueba (considerado caso base), por lo que podemos concluir, que este índice también detecta los elementos de transmisión más débiles, respecto a la estabilidad de voltaje. 


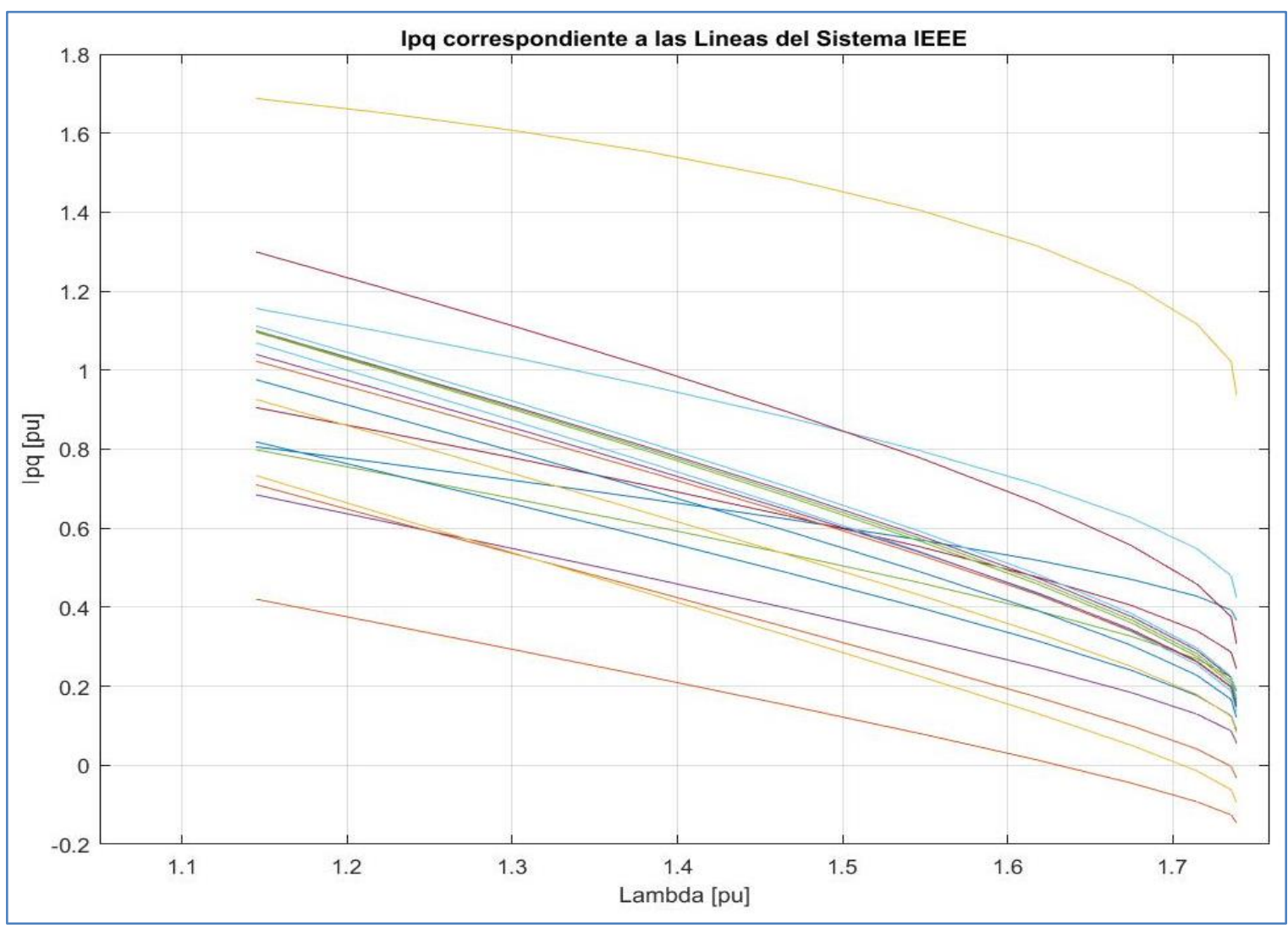

Figura 6 Comportamiento del índice de estabilidad de tensión Ipq para el Sistema

IEEE 14 de Barras

\section{CONCLUSIÓN O CONSIDERACIONES FINALES}

- El método de flujo de potencia continuado mediante el método de parametrización geométrica, ayuda a determinar la estabilidad del sistema eléctrico de potencia, incluyendo la máxima demanda que pueda soportar el sistema antes de llegar a la inestabilidad de voltaje.

- Respecto a los índices basados en los niveles de voltajes en las barras, solamente determina, aquellas barras que se encontraran más cercanos a la inestabilidad de voltaje, llegando alcanzar una curvatura, que demuestra que el sistema ya no puede soportar más demanda y se llegó a la inestabilidad de voltaje.

- Respecto a los índices de estabilidad, basados en las perdidas de potencia activa y reactiva, se tiene un comportamiento exponencial creciente, donde los elementos de transmisión que producen mayor cantidad de pérdidas son considerados más propensos a contribuir a la inestabilidad de voltaje, pero también se debe tener en consideración, que estos elementos pueden ser robustos (mayor capacidad de 
transmisión de energía), por las cuales podrán soportar mayor cantidad de perdidas, esto significa que dicho índice no ofrece información completa.

- Respecto al índice de estabilidad, basados en los flujos de potencia activa y reactiva Ipq, tiene mayor ventaja en la detección de aquellos elementos de transmisión que son más débiles o que tienen una mayor contribución a la inestabilidad de voltaje, debido a que en un solo sistema de ecuaciones, engloba a la cantidad de potencia activa y reactiva, produciendo que el esfuerzo computacional sea menor y con un mejor, teniendo en cuenta, que aquellos elementos que tengan menores magnitudes, serán más sensibles a cambios súbitos de la demanda

\section{LISTA DE REFERENCIAS}

B. Gao; G. K. Morison; P. Kundur, "Voltage Stability Evaluation Using Modal Analysis", IEEE Transactions on Power System, Vol. 7, No 4, pp. 1529 - 1541, November 1992.

A.Sode-Yome, N.Mithulananthan, and K.Y.Lee, "Effect of realistic load direction in static voltage stability study," in IEEE/PES Transmission and Distribution Conference \& Exposition: Asia \& Pacific, 2005

N.Amjady and M.Esmaili, “Application of new sensitivity analysis framework for voltage contingency ranking," IEEE Trans. Power Systems, Vol.20, No.2, pp. 973-983, 2005

V. Ajjarapu, B. Lee, "Bibliography on Voltage Stability", IEEE Trans. On Power Systems, Vol. 13, No 1, pp.115-125, February 1998.

V. Ajjarapu, “Computational Techniques for Voltage stability Assessment and Control”, 2da Edición, Estados Unidos, Springer, pp. 250, 2006.

Grainger, J., Stevenson, W. “Análisis de Sistemas de Potencia”, 3ra Edición, México, McGraw Hill, 1996, pp. 561

Gómez, A. E. “Análisis y Operación de Sistemas de Energía Eléctrica”, 2da Edición, Madrid, McGraw Hill, 2002, pp. 769

Bonini, A. N. (2006), Proposición de una Técnica de Parametrización Geométrica para el Flujo de Carga Continua Basado en las Variables de Tensión y Carga [en línea]. Tesis de Maestría. Universidad Estatal Paulista, Brasil 
Domingues, S. B. (2008). Métodos de Análisis de Sensibilidad para la Evaluación de la Estabilidad de Voltaje de Sistemas Eléctricos de Potencia [en línea]. Tesis de Maestría. Instituto Politécnico Nacional, México.

Gamboa, W., Plazarte, J. (2010). Análisis de Estabilidad de Voltaje Utilizando Análisis Modal [en línea], CENACE, Ecuador

Klump, R., Overbye, Th. (2000), Techniques for Improving Power Flow Convergence [en linea], IEEE 2000, Power World Corporation

Lázaro, M. G. (2005). Análisis de Estabilidad ante Pequeños Disturbios de los Generadores de Inducción en Sistemas Eléctricos de Potencia [en línea], Tesis de Maestría. Instituto Politécnico Nacional, México

Lof, A. P., Smed, T., Andersson, G. (1992), Fast Calculation of a Voltage Stability Index [en linea], IEEE transactions on Power Systems

Lopez, E. L. (2006). Análisis de la Estabilidad de Voltaje de Sistemas Eléctricos de Potencia Utilizando el Método de Flujos de Potencia de Continuación [en línea]. Tesis de Maestría. Instituto Politécnico Nacional, México

Mello, E. M. (2010), Aplicacao do Método de Newton Desacoplado para o Fluxo de Carga Continuado [en línea]. Tesis de Maestría, UNESP, Brazil.

Monticelli, A. J., (1983). 1ra Edición, Brasil. Fluxo de Carga en Redes de Energia Eléctrica. EDITORA EDGARD BLUCHER Ltda. PP 164

Ortega O.N., Quezada, A. E., Herrera, R. E. (2009), Flow a Tool for Analysis of Power Flow and Facts Devices [en línea], ISSN: 1692-7275, Revista Colombiana de Tecnologías de Avanzadas, Colombia

Samaniego, E. M. R. (2013), Detección de Áreas Débiles respecto a la Estabilidad de Tensión en Tiempo Real utilizando Lógica Difusa [en línea], Tesis de Maestría, Universidad de Cuenca, Ecuador.

Santillana, A. (2005). Determinación de Puntos Críticos para el Análisis de Estabilidad de Voltaje en Sistemas Eléctricos de Potencia [en línea], Tesis de Maestra. Instituto Politécnico Nacional, México.

Sharma, Ch. (2007), Determination of Power System Voltage [en linea] IEEE POWERENG 